\title{
Protective effects of resveratrol on acute kidney injury in rats with sepsis
}

\author{
Cong-Juan Luo, Feng Luo, Quan-Dong Bu, Wei Jiang, Wei Zhang, Xue-Mei Liu, Lin Che, Hong Luan, Hui Zhang, Rui-Xia Ma, \\ Jian-Ping Sun, Yan Xu
}

\begin{abstract}
Aim. To evaluate the protective effects of resveratrol on acute kidney injury (AKI) in septic rats.
Methods. A septic rat model was established by cecal ligation and puncture (CLP). A total of 108 male Sprague Dawley rats were randomly divided into an observation group, a $6 \mathrm{~h}$ resveratrol intervention group and a $12 \mathrm{~h}$ resveratrol intervention group. Then each group was subdivided into Sham, Sham + Res, CLP and CLP + Res groups. After surgery, the survival and morphological changes in kidney tissues were observed. Serum creatinine and urea nitrogen levels, expression of GRP78, BiP, IRE1 and p65 in kidney tissues, and serum levels of TNF- $\alpha$, IL-1 $\beta$, IL- 6 and IL-10 were investigated. Results. The survival rate of CLP + Res group (75.00\%) significantly exceeded that of the CLP group (41.67\%) $(P<0.05)$. At postoperative $12 \mathrm{~h}$, resveratrol significantly decreased serum creatinine and urea nitrogen levels $(P<0.05)$. Resveratrol evidently relieved renal tubular swelling and luminal narrowing in CLP rats, and significantly reduced the high expressions of GRP78, BiP, phosphorylated IRE1 and p65 proteins $(P<0.05)$. P65 was mainly located in the cytoplasm of Sham, Sham + Res and CLP + Res groups, and in the nucleus of the CLP group. At postoperative $12 \mathrm{~h}$, resveratrol significantly reduced serum levels TNF-a, IL-1 $\beta$ and IL- 6 in CLP rats $(P<0.05)$, whereas elevated that of IL-10 $(P<0.05)$.

Conclusion. Resveratrol significantly decreased the mortality rate of septic rats and alleviated AKI, probably by attenuating endoplasmic reticulum stress, inhibiting activation of the NF-KB pathway and mitigating the inflammatory response.
\end{abstract}

Key words: resveratrol, sepsis, acute kidney injury, endoplasmic reticulum stress, inflammatory response

Received: September 12, 2018; Accepted with revision: February 26, 2019; Available online: April 17, 2019

https://doi.org/10.5507/bp.2019.006

(c) 2020 The Authors; https://creativecommons.org/licenses/by/4.0/

The Affiliated Hospital of Qingdao University, No.16 Jiangsu Road, Qingdao 266003, Shandong Province, China Corresponding authors: Feng Luo, e-mail: Iuofengahqu@hotmail.com; Yan Xu, e-mail:xuyanahqu@hotmail.com

\section{INTRODUCTION}

Sepsis is a systemic inflammatory response syndrome caused by infection, which often occurs after severe trauma, burn and major surgery, leading to multiple organ dysfunction syndrome and even death due to uncontrollable inflammation ${ }^{1}$. The incidence rate of sepsis is high, and the treatment outcomes are unsatisfactory ${ }^{2}$. Over 18 million patients with severe sepsis worldwide each year usually die from related complications. Although the mechanism of sepsis has been widely studied, the mortality rate is still as high as $30-70 \%$, and is mainly responsible for the death of patients in intensive care units. The kidney is one of the organs most susceptible to sepsis. The incidence rates of acute renal injury (AKI) in patients with mild, moderate and severe sepsis are 19\%,23\% and $51 \%$ respectively. In case of acute renal failure, the mortality rate can even reach up to $70 \%$ (ref. ${ }^{3,4}$ ). Therefore, early prevention and treatment of AKI have great clinical significance for reducing the mortality rate of sepsis and improving the prognosis.

At present, the mechanism of AKI during sepsis has not been fully elucidated. The production and release of inflammatory mediators in sepsis, changes in renal hemodynamics, abnormal blood coagulation, as well as immune-induced injury and apoptosis are all involved in the onset of AKI, and uncontrollable waterfall-like cascade of inflammatory mediators also plays an essential role ${ }^{5}$.
Inflammatory cytokines TNF- $\alpha$, IL-1 $\beta$, IL-6 and IL-10 are mainly associated with AKI during sepsis ${ }^{6-9}$. Nuclear transcription factor- $\mathrm{KB}(\mathrm{NF}-\mathrm{\kappa B})$ is a key transcriptional regulator of inflammation-related genes. After activation, it can induce transcriptional synthesis of inflammatory factors, and trigger a waterfall-like cascade of inflammatory mediators, thereby predominantly participating in the onset and progression of sepsis ${ }^{10}$.

The activity of NF- $\mathrm{kB}$ is regulated by inositol requiring enzyme 1 (IRE1) located on the endoplasmic reticulum membrane. The endoplasmic reticulum is a crucial organelle for protein synthesis, folding and secretion in eukaryotic cells. With a dynamic membrane structure, it is sensitive to various external stimuli. Infection, trauma, burn and major surgery can induce endoplasmic reticulum stress (ERS). Under ERS, IRE1 is activated by autophosphorylation, followed by binding and activation of IKK, then regulating the transcription of various inflammatory factors. ERS also plays an important role in the onset and progression of sepsis ${ }^{11}$. Esposito et al. found that lipopolysaccharide (LPS)-induced AKI was closely related to ERS (ref. ${ }^{12}$ ). Besides, Calisto et al. reported that ERS responses in the liver, fat and skeletal muscle of a septic rat model established by cecal ligation and puncture (CLP) were significantly enhanced ${ }^{13}$. In addition, Ma et al. found that lymphocyte apoptosis during sepsis was associated with ERS-induced accumulation of spliced XBP1 mRNA and up-regulation of CHOP protein 
and mRNA expressions ${ }^{14}$. Therefore, we hypothesized that ERS-induced increase in NF- $\mathrm{B}$ activity and release of considerable inflammatory factors may dominate in the onset and progression of AKI during sepsis.

As a polyphenolic compound with the chemical name 3,4,5-trihydroxystilbene, resveratrol has received extensive attention owing to anti-inflammatory, antitumor, cardiovascular protective and other beneficial biological activities ${ }^{15}$. It has protective effects on sepsis-induced acute lung injury and myocardial depression ${ }^{16}$. Moreover, resveratrol can alleviate insulin resistance, inhibit retinal degeneration and combat tumors by suppressing ERS (ref. ${ }^{17}$ ). Holthoff et al. found that administration of resveratrol after CLP improved renal microcirculation, reduced reactive nitrogen species, and further protected renal tubular epithelial cells ${ }^{18}$. However, whether resveratrol can protect against AKI upon sepsis remains largely unknown.

Based on the above literatures, we postulated that resveratrol would be able to protect against the onset and progression of AKI in the case of sepsis, probably related to the ERS-IRE1-NF- $\mathrm{B}$ pathway. To confirm this hypothesis, a rat model of sepsis was established by CLP. The effects of resveratrol on the survival rate and time, renal function, renal tissue morphology, expressions of ERS marker proteins, p65 and inflammatory cytokines in renal tissues, as well as serum inflammatory cytokine levels were evaluated, aiming to provide valuable evidence for the clinical prevention and treatment of AKI during sepsis.

\section{MATERIALS AND METHODS}

\section{Experimental animals}

Animal experiments were by the ethics committee of The Affiliated Hospital of Qingdao University, and great efforts were made to minimize animal suffering. A total of 108 healthy male Sprague Dawley rats weighing 200250 g (permission number: cSCXK (Jiangsu Province) 2014-0007) were purchased from Suzhou Industrial Park Ai'er Maite Technology Co., Ltd. (China). The rats were fed in a clean and quiet environment at $18-21^{\circ} \mathrm{C}$ with a relative humidity of $40 \%-60 \%$. In a $12 \mathrm{~h} / 12 \mathrm{~h}$ light-dark cycle, they were fed routinely, with free access to water. Three rats were kept in a plastic cage. After one week of adaptive feeding, the rats with normal status were included in the study.

\section{Main antibodies and reagents}

Resveratrol (R5010) was purchased from Sigma (USA). The BCA kit was bought from Beijing Dingguo Changsheng Biotechnology Co., Ltd. (China). DAB color development solution, HRP-labeled goat anti-mouse IgG and goat anti-rabbit IgG antibodies were obtained from Wuhan Boster Biological Technology Co., Ltd. (China). Rat TNF- $\alpha$, IL-6, IL-10 and IL-1 $\beta$ detection kits were provided by Hangzhou Lianhe Biotechnology Co., Ltd. (China). Rabbit anti-GRP78/BiP polyclonal antibody (ADI-SPA-826) was supplied by Stressgen (USA). Rabbit
anti-IRE1 (ab48187) and anti-p65 polyclonal antibodies (ab7970) were bought from Abcam (USA).

\section{Establishment of septic rat model}

A rat model of sepsis was established through CLP. In detail, rats were intraperitoneally injected with $30 \mathrm{mg} / \mathrm{kg}$ $10 \%$ chloral hydrate, and the abdomen skin was sterilized by iodophor, with an approximately 2-3 incision made at the midline. The mesentery and cecum were disconnected, and about $1 \mathrm{~cm}$ from the dead end of the cecum was ligated with 4\# silk thread. Finally, the abdomen was closed layer-by-layer.

\section{Preparation of resveratrol solution}

Resveratrol is easily soluble in organic solutions but not water, also sensitive to light. Detailedly, $1.8 \mathrm{~g}$ resveratrol was completely dissolved with $2 \mathrm{~mL}$ of $100 \%$ DMSO, and supplemented with DMSO into $3 \mathrm{~mL}$. Afterwards, $0.5 \mathrm{~mL}$ of the solution was added into $150 \mathrm{~mL}$ normal saline. The final concentration of resveratrol was $2 \mathrm{mg} / \mathrm{mL}$.

\section{Animal grouping}

The 108 rats were randomly divided into an observation group (group A), a $6 \mathrm{~h}$ resveratrol intervention group (group B) and a $12 \mathrm{~h}$ resveratrol intervention group (group C). Then each group was subdivided into a Sham group receiving sham surgery and postoperative intraperitoneal injection with $15 \mathrm{~mL} / \mathrm{kg}$ normal saline containing $0.33 \%$ DMSO, a Sham + Res group receiving sham surgery and postoperative intraperitoneal injection with $30 \mathrm{mg} / \mathrm{kg}$ resveratrol, a CLP group receiving intraperitoneal injection with $15 \mathrm{~mL} / \mathrm{kg}$ normal saline containing 0.33\% DMSO after modeling, and a CLP + Res group receiving intraperitoneal injection with $30 \mathrm{mg} / \mathrm{kg}$ resveratrol after modeling. After surgery, all rats were given free access to water and feed. Each subgroup of group A comprised 15 rats, and each subgroup of groups $\mathrm{B}$ and $\mathrm{C}$ consisted of 6 rats.

\section{Sample collection and preparation}

Timing was started immediately after postoperative suturing of the abdominal wall, and rats were killed at indicated time points. Blood $(2 \mathrm{~mL})$ was collected from the heart, and centrifuged at $4{ }^{\circ} \mathrm{C}$ and $1000 \times \mathrm{g}$ for $15 \mathrm{~min}$. The resulting serum was collected and stored in a $-80{ }^{\circ} \mathrm{C}$ refrigerator. Then serum creatinine and urea nitrogen levels were measured with an automatic biochemical analyzer. The left kidney was taken, from which surface fat and fascia were peeled off. The residue was added into EP tubes, immediately put in liquid nitrogen, and stored in the $-80{ }^{\circ} \mathrm{C}$ refrigerator. The right kidney was taken, transversely incised in the center, fixed in $10 \%$ formalin, and embedded with paraffin to prepare sections.

\section{Western blotting}

Total protein was extracted from $0.1 \mathrm{~g}$ kidney tissue by adding $1 \mathrm{~mL}$ of lysate. Protein concentration was detected by BCA kit. After electrophoresis, the proteins were electronically transferred onto a PVDF membrane at $4{ }^{\circ} \mathrm{C}$. 
Subsequently, the membrane was blocked with $5 \%$ BSA for $2 \mathrm{~h}$, incubated with corresponding primary antibodies overnight at $4{ }^{\circ} \mathrm{C}$, washed, incubated with secondary antibodies at $37^{\circ} \mathrm{C}$ for $1 \mathrm{~h}$, developed with ECL reagent and scanned. Relative protein expression was analyzed by Quantity One software after internal reference correction using GAPDH.

\section{ELISA}

Diluted sample, standard and control solutions (50 $\mu \mathrm{L}$ each) were added into each well of 6-well plates. Then detection antibody $(50 \mu \mathrm{L})$ was added into each well. Afterwards, the plate was sealed, shaken at $100 \mathrm{rpm}$ and incubated at room temperature for $3 \mathrm{~h}$. After the liquid was discarded, each well was washed with $300 \mu \mathrm{L}$ of washing solution, 6 times in total. Subsequently, 100 $\mu \mathrm{L}$ of HRP-streptavidin conjugate was added into each well, and the plate was sealed, shaken at $100 \mathrm{rpm}$ and incubated at room temperature for $45 \mathrm{~min}$. After the liquid was discarded, each well was washed again with $300 \mu \mathrm{L}$ of washing solution, 6 times in total. TMB $(100 \mu \mathrm{L})$ was added into each well for another 10-30 min of incubation in dark, and then $100 \mu \mathrm{L}$ of stopping buffer was added into each well. The optical densities at $450 \mathrm{~nm}$ and 630 $\mathrm{nm}$ were thereafter measured with a microplate reader within $30 \mathrm{~min}$. Serum levels of related factors or proteins were detected.

\section{Immunohistochemical assay}

Sections were deparaffinized at $58{ }^{\circ} \mathrm{C}$ for $30 \mathrm{~min}$ using xylene three times ( 5 min each time), $100 \%$ ethanol three times ( 5 min each time), 95\% ethanol solution twice (5 min each time), 80\% ethanol solution for $3 \mathrm{~min}, 70 \%$ ethanol solution for $3 \mathrm{~min}$ and distilled water for $10 \mathrm{~min}$. After antigen retrieval using $\mathrm{pH} 6.0$ citrate buffer at $80^{\circ} \mathrm{C}$ for $20 \mathrm{~min}$, the sections were cooled down to room temperature, and washed twice with PBS ( 5 min each time). Subsequently, they were blocked with $1 \%$ bovine serum albumin (BSA), incubated with anti-p65 primary antibody (1:25 diluted by $1 \% \mathrm{BSA}$ ) at $4{ }^{\circ} \mathrm{C}$ overnight, washed three times with PBS ( 5 min each time), incubated with HRPlabeled rabbit secondary antibody (diluted by $1 \%$ BSAPBS) for $1 \mathrm{~h}$ at room temperature, washed again with PBS ( 5 min each time), and color-developed with DAB solution for 1-2 min. After the reaction was stopped by adding tap water, the sections were washed with PBS three times (10 min each time), stained with hematoxylin and eosin (H\&E) for 1-3 min, and washed with $100 \%$ ethanol three times ( 5 min each time) and xylene twice ( 5 min each time). Finally, they were mounted with neutral resin and observed under an optical microscope.

\section{Statistical analysis}

All data were analyzed by SPSS16.0 software. The categorical data were expressed as frequencies. Intergroup comparisons were performed by one-way analysis of variance, and pairwise comparisons were conducted with the SNK-q test. The numerical data were expressed as percentage, and inter-group comparisons were carried out by the $\chi^{2}$ test. Survival was analyzed by the log-rank test. $P<0.05$ was considered statistically significant.

\section{RESULTS}

\section{Effects of resveratrol on general symptoms and tissue damages of septic rats}

With or without resveratrol treatment, the Sham group was free from obvious abnormalities after surgery. In contrast, the CLP group suffered from apparent symptoms and tissue damage which were relieved in the CLP + Res group.

\section{Effects of resveratrol on survival rates of septic rats}

The survival rates of Sham and Sham + Res groups at $72 \mathrm{~h}$ were $100 \%$, significantly surpassing that of the CLP group (41.67\%) $(P<0.05)$ (Fig. 1). The survival rate of the CLP + Res group $(75.00 \%)$ was significantly higher than that of the CLP group $(P<0.05)$.

\section{Effects of resveratrol on serum creatinine and urea nitrogen levels of septic rats}

At postoperative $12 \mathrm{~h}$, the serum creatinine and urea nitrogen levels of the CLP group were significantly higher than those of the Sham group $(P<0.05)$, which were significantly decreased by resveratrol treatment $(P<0.05)$ (Fig. 2).

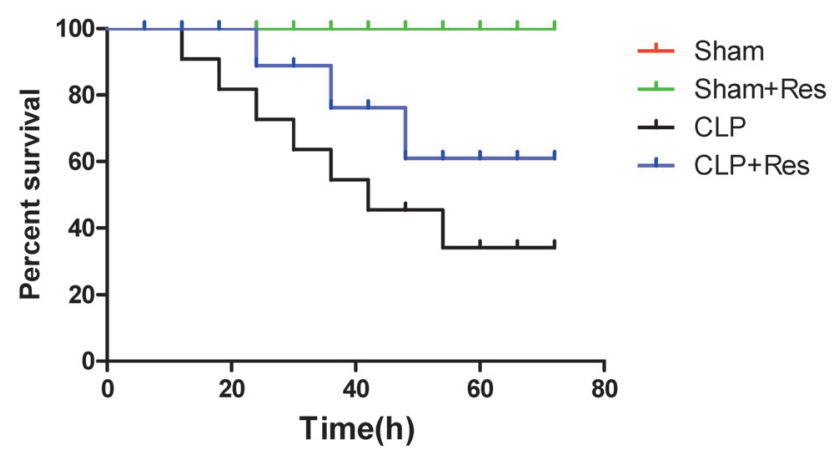

Fig. 1. Effects of resveratrol on survival rates of septic rats.
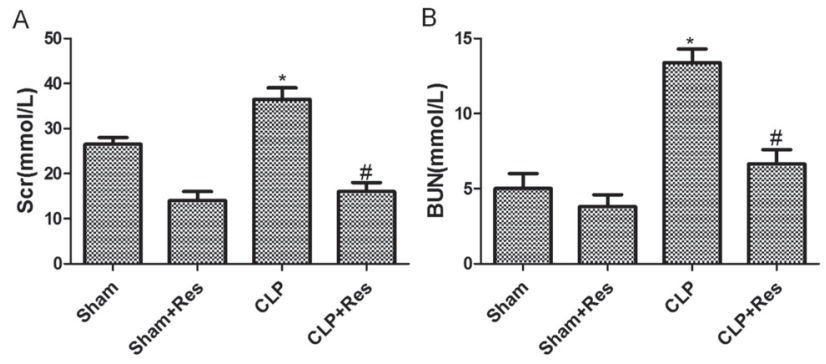

Fig. 2. Effects of resveratrol on (A) serum creatinine and (B) urea nitrogen levels of septic rats. ${ }^{*}$ Compared with Sham group, $P<0.05$; \#compared with CLP group, $P<0.05$. 

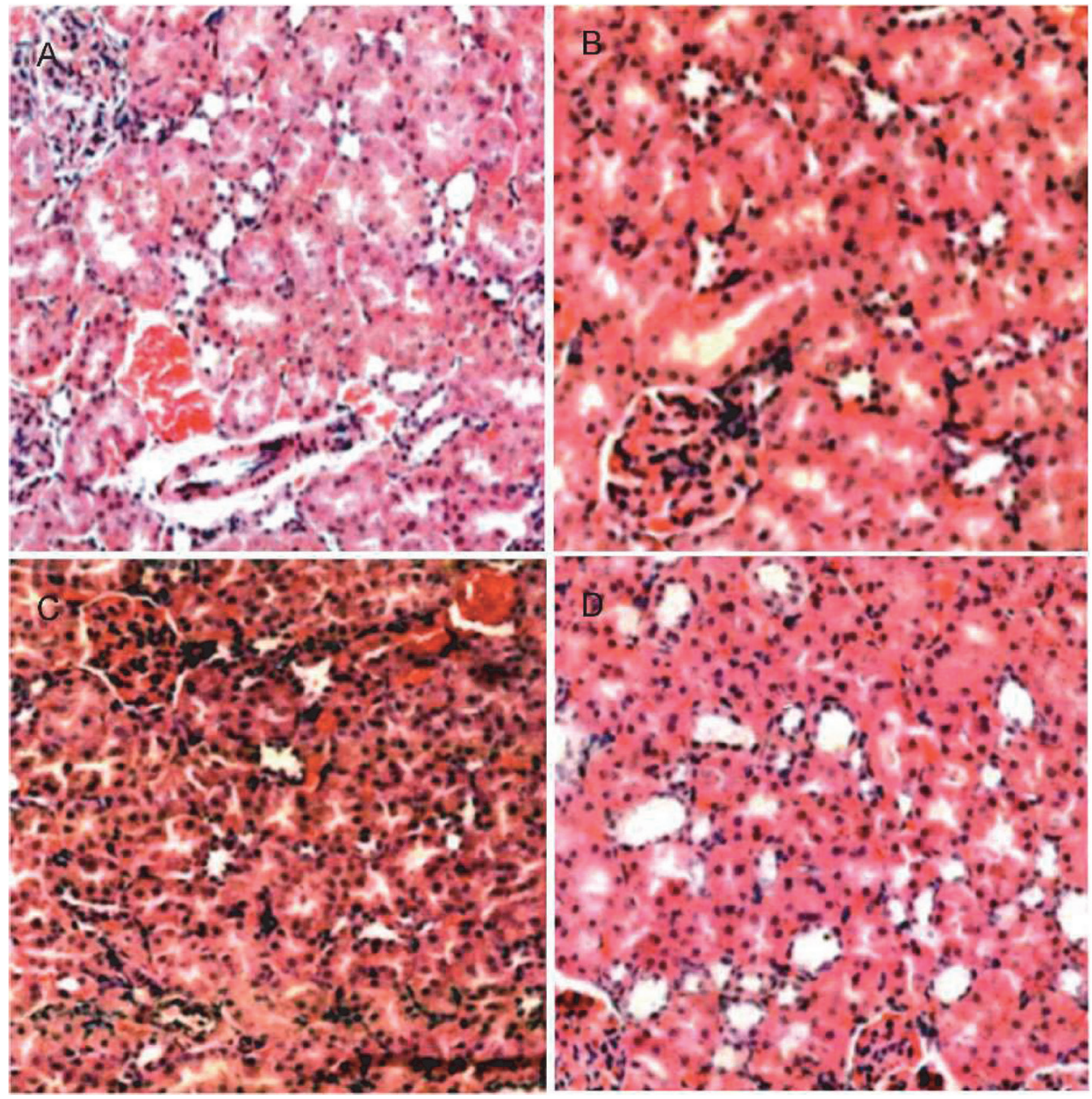

Fig. 3. H\&E staining results of kidney tissue sections.

A: Sham group; B: Sham + Res group; C: CLP group; D: CLP + Res group.

Effects of resveratrol on kidney tissue morphologies of septic rats

H\&E staining of kidney tissue sections showed that the renal glomerular morphologies of all groups were similar. Sham and Sham + Res groups had normal tubular morphologies and obvious lumens, whereas the CLP group underwent evident renal tubular swelling and luminal narrowing which were markedly attenuated by resveratrol treatment (Fig. 3). Collectively, resveratrol relieved sepsis-induced AKI.

\section{Effects of resveratrol on BiP and phosphorylated IRE1} protein expressions in kidney tissues of septic rats

Western blot exhibited that significantly more BiP and phosphorylated IRE1 were expressed in the kidney tissue of the CLP group than in the Sham group $(P<0.05)$. Compared with the CLP group, both expressions in the CLP + Res group significantly decreased $(P<0.05)$ (Fig. 4). Therefore, resveratrol significantly attenuated ERS in damaged kidney tissue.
Effects of resveratrol on p65 protein expressions in kidney tissues of septic rats

Immunohistochemical assay displayed that $\mathrm{p} 65$ was mainly located in the cytoplasm of Sham, Sham + Res and CLP + Res groups, while in the nucleus of the CLP group (Fig. 5A-D). The brown particle represents positively expressed $\mathrm{p} 65$ protein. The positive expression rate of p65 in the CLP group significantly exceeded those of Sham and Sham + Res groups, and the rate of the CLP + Res group was significantly lower than that of the CLP group $(P<0.05)$ (Fig. 5E).

Effects of resveratrol on serum TNF- $\alpha$, IL-1 $\beta$, IL-6 and IL-10 levels in septic rats

Six hours after surgery, resveratrol significantly decreased serum TNF- $\alpha$, IL-1 $\beta$ and IL-6 levels $(P<0.05)$ (Fig. 6A-C) but not that of IL-10 in CLP rats $(P>0.05)$ (Fig. 6D).

At postoperative $12 \mathrm{~h}$, it significantly reduced serum levels TNF- $\alpha$, IL-1 $\beta$ and IL-6 in CLP rats $(P<0.05)$ (Fig. 7A-C), whereas elevated that of IL-10 $(P<0.05)$ (Fig. 7D). 

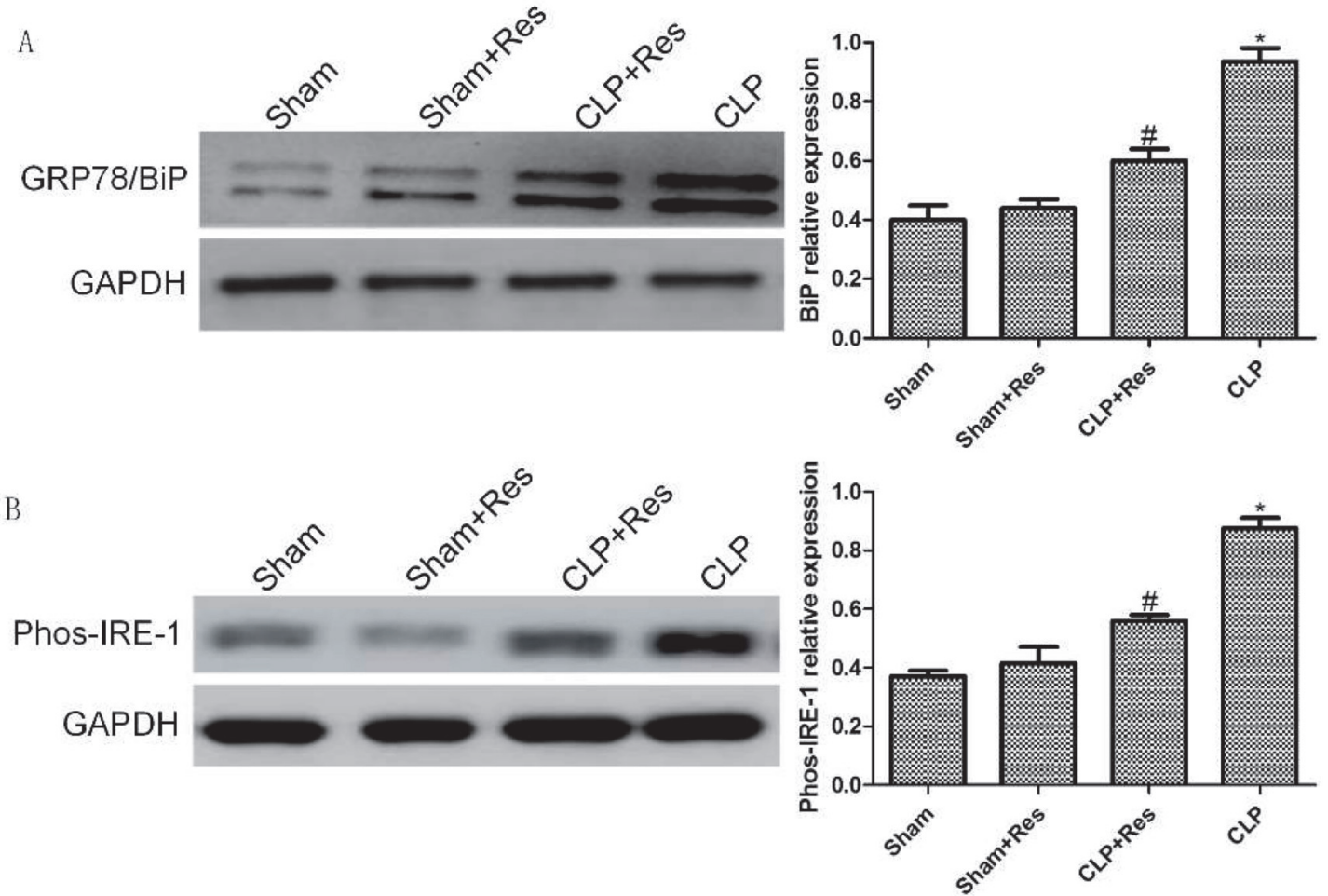

Fig. 4. Effects of resveratrol on (A) BiP and (B) phosphorylated IRE1 protein expressions in kidney tissues of septic rats. *Compared with Sham group, $P<0.05$; \#compared with CLP group, $P<0.05$.

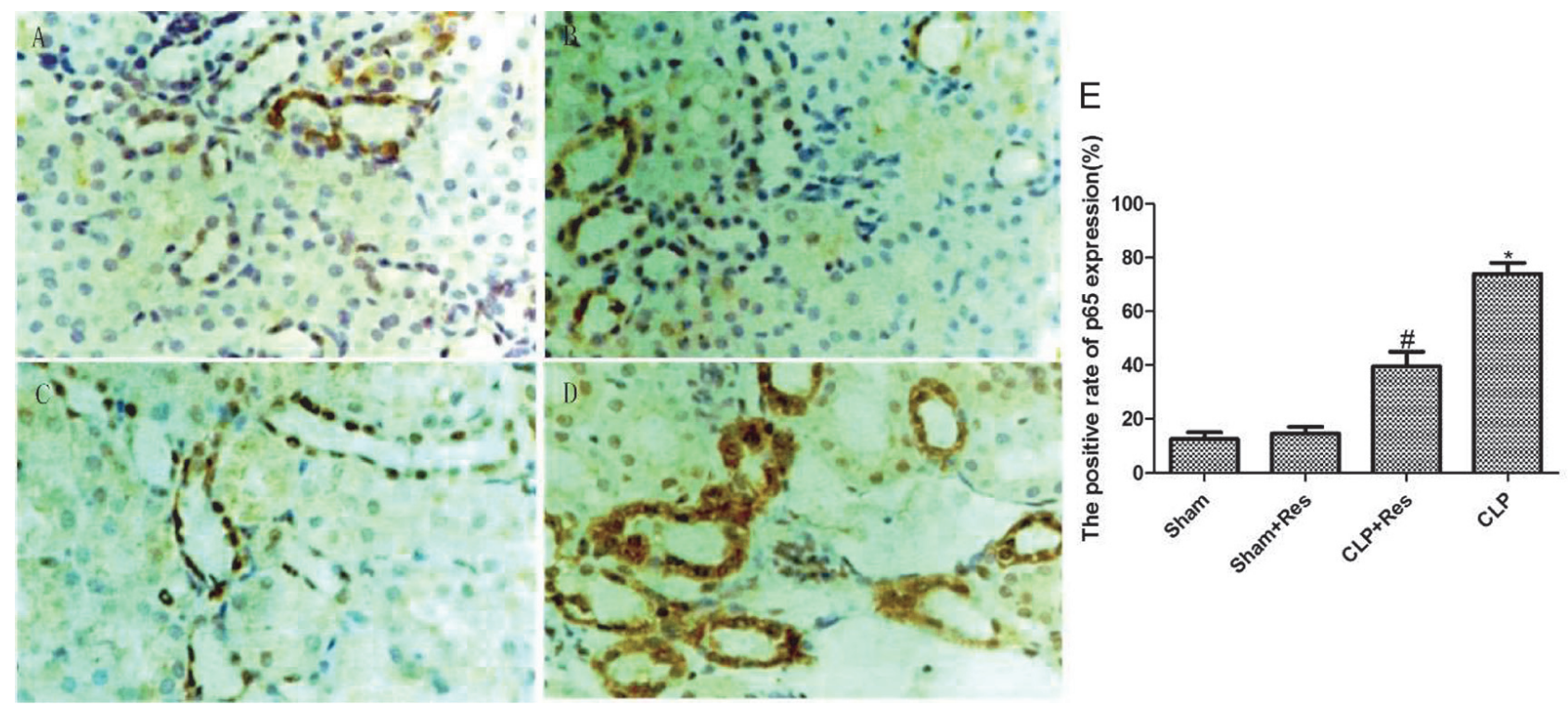

Fig. 5. Expressions and locations of $\mathrm{p} 65$ in kidney tissues of septic rats $(\times 400)$.

A: Sham group; B: Sham + Res group; C: CLP + Res group; D: CLP group; E: positive expression rates. *Compared with Sham group, $P<0.05$; \#compared with CLP group, $P<0.05$.

\section{DISCUSSION}

Currently, sepsis is one of the critical problems for emergency medicine ${ }^{19}$, and the mechanisms for sepsisinduced AKI remain largely unknown. ERS in kidney tissues has been reported to mediate the onset of kidney injury. For instance, Gao et al. found that ERS participated in ischemia-reperfusion-induced AKI, and inhibition of ERS significantly alleviated the resulting renal tubular necrosis and cell apoptosis ${ }^{20}$. Besides this, ERS promotes renal glomerular and tubular injury in patients with different acute and chronic kidney diseases ${ }^{21}$.

In this study, we first established a rat model of sepsis through CLP. As a result, the serum creatinine and urea nitrogen levels significantly increased, accompanied by kidney dysfunction and moderate morphological damages manifested as tubular epithelial cell swelling and luminal narrowing. The results may be related with the structural characteristics of the kidney. Similarly, Tran et al. constructed a model of endotoxemia-induced AKI through 

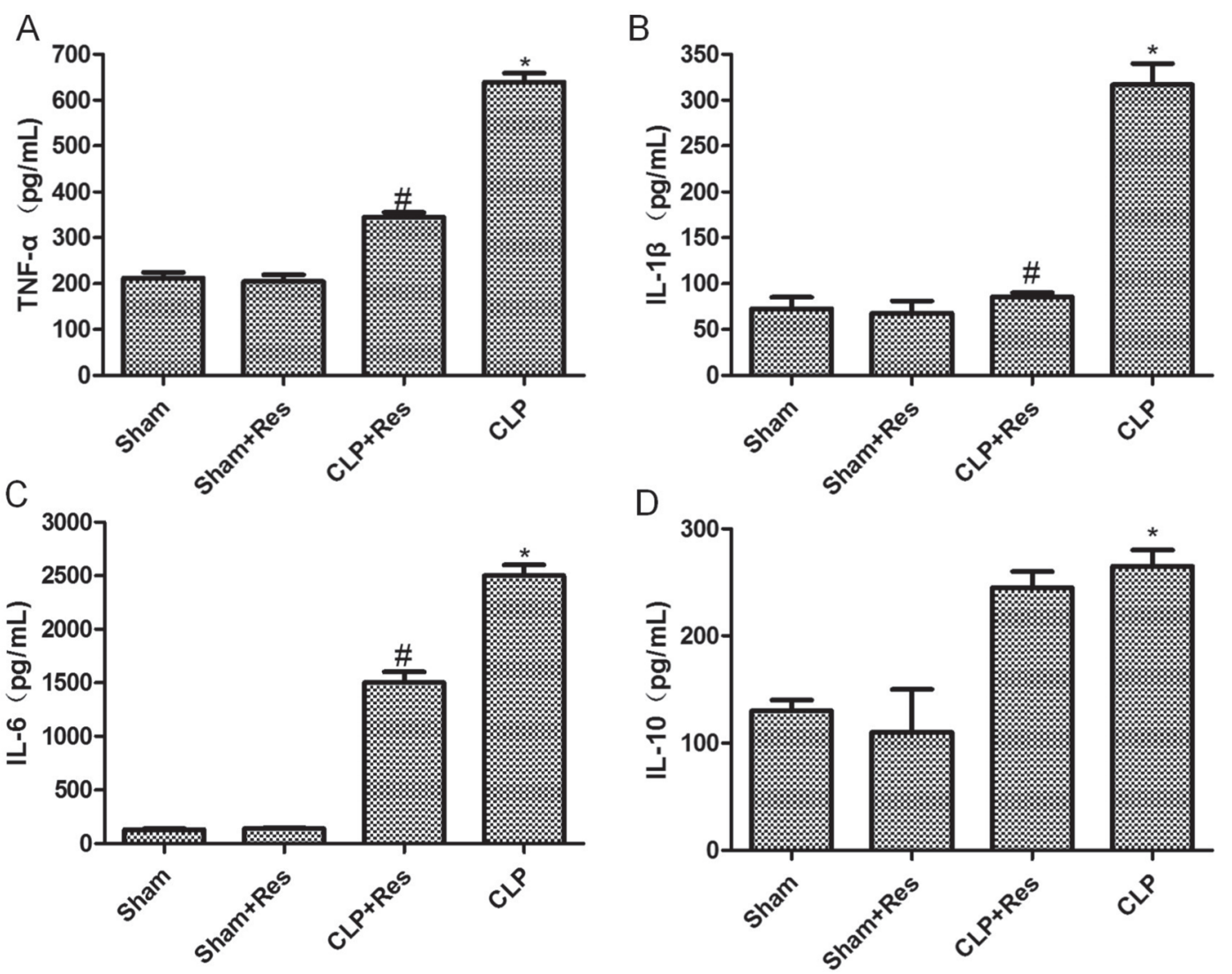

Fig. 6. Effects of resveratrol on serum (A) TNF- $\alpha$, (B) IL-1 $\beta$, (C) IL-6 and (D) IL-10 levels in septic rats $6 \mathrm{~h}$ after surgery. ${ }^{*}$ Compared with Sham group, $P<0.05$; \#compared with CLP group, $P<0.05$.
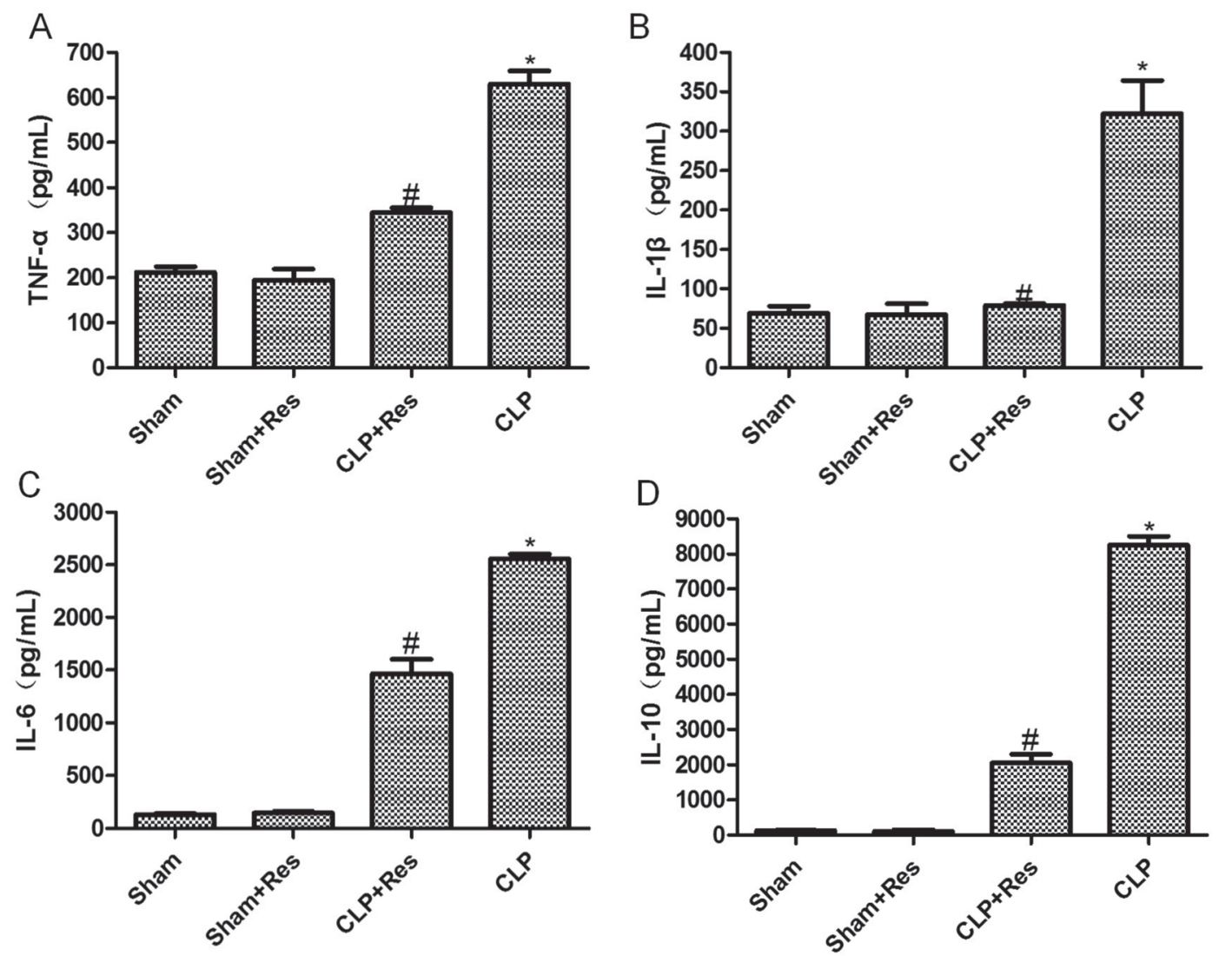

Fig. 7. Effects of resveratrol on serum (A) TNF- $\alpha$, (B) IL-1 $\beta$, (C) IL-6 and (D) IL-10 levels in septic rats $12 \mathrm{~h}$ after surgery. *Compared with Sham group, $P<0.05$; \#compared with CLP group, $P<0.05$. 
intraperitoneal injection of LPS, and found that fewer than $10 \%$ of proximal renal tubular cells underwent vacuolar degeneration and local necrosis without apoptosis ${ }^{22}$. In addition, we detected the expressions of ERS marker proteins (IRE1 and GRP78), the main subunit of NF-kB (p65) as well as related inflammatory cytokines TNF- $\alpha$, IL-1 $\beta$, IL-6 and IL-10. Given that the expressions of IRE1, GRP78 and p65 were up-regulated and inflammatory response was enhanced, "ERS-IRE1-NF-kB-inflammatory response" may be closely associated with AKI during sepsis.

Jung et al. reported that resveratrol or its tetramer vaticanol B attenuated insulin resistance and inflammatory factor expression by inhibiting ERS, and also suppressed IL-1 $\beta$ - and LPS-mediated NF- $\mathrm{kB}$ activation and transcriptional activity, thereby down-regulating the expressions of target genes such as TNF- $\alpha$, IL-6, IL-1 $\beta$ and iNOS (ref. ${ }^{23}$ ). Here, administration with resveratrol significantly reduced the mortality rate of CLP rats, improved their renal functions, and inhibited the swelling of renal tubular epithelial cells. Likewise, Holthoff et al. found that treatment with resveratrol after CLP improved the renal microcirculation, eliminated nitrogen free radicals, protected renal tubular epithelial cells, and thus improved the survival rate of rats with sepsis-induced AKI (ref. ${ }^{18}$ ). Accordingly, resveratrol can markedly protect against CLP-induced AKI of septic rats, mainly by improving the renal functions at the early stage. Moreover, resveratrol significantly reduced ERS and p65 expression in kidney tissue. Meanwhile, it decreased the expressions of serum TNF- $\alpha$, IL-1 $\beta$ and IL- 6 six hours after CLP, but hardly affected that of IL-10. At postoperative $12 \mathrm{~h}$, resveratrol significantly down-regulated serum TNF- $\alpha$, IL- $1 \beta$ and IL- 6 expressions, whereas it significantly up-regulated that of IL-10. Possibly, considerable pro-inflammatory cytokines were released with the progression of inflammation, and resveratrol exerted potent anti-inflammatory effects by both suppressing the expressions of pro-inflammatory cytokines and facilitating those of anti-inflammatory ones.

\section{CONCLUSION}

In summary, we verified that resveratrol protected against sepsis-induced AKI, which may be linked to inhibition of the "ERS-IRE1-NF-kB-inflammatory response" pathway and mitigation of inflammatory response in kidney tissue. Regardless, further in-depth studies are still in need to clarify whether resveratrol inhibits ERS by playing its well-known antioxidant role.

Acknowledgement: This study was supported by grants from National Natural Science Foundation of China (No. 81700585, 81170688, 81470973, 81770679).

Author contribution: All authors contributed equally to preparing the manuscript.

Conflict of interest statement: None declared.

\section{REFERENCES}

1. Kaukonen KM, Bailey M, Pilcher D, Cooper DJ, Bellomo R. Systemic inflammatory response syndrome criteria in defining severe sepsis. N Engl J Med 2015;372(17):1629-38.

2. Zhong W, Leto $G$, Wang $L$, Zeng G. Systemic inflammatory response syndrome after flexible ureteroscopic lithotripsy: a study of risk factors. J Endourol 2015;29(1):25-8.

3. Delmas C, Zapetskaia T, Conil JM, Georges B, Vardon-Bounes F, Seguin T, Crognier L2, Fourcade O, Brouchet L, Minville V, Silva S. 3-month prognostic impact of severe acute renal failure under veno-venous ECMO support: Importance of time of onset. J Crit Care 2018;44:63-71.

4. Schmitz M, Tillmann FP, Paluckaite A, Laufer EA, Rayner B, Rump LC, Heering PJ. Mortality risk factors in intensive care unit patients with acute kidney injury requiring renal replacement therapy: a retrospective cohort study. Clin Nephrol 2017;88(1):27-32.

5. He T, Tao J, Wang X, Wang X. Effects of cisatracurium in combination with ventilation on inflammatory factors and immune variations in sepsis rats. Exp Ther Med 2018;15(5):4414-8.

6. Peng N, Geng Y, Zhang S, Su L. Effects of ulinastatin on oxidative stress and renal injury in rats with severe heatstroke. Med J Chin People Liber Army 2015;40(5):354-7.

7. Szeto HH, Liu S, Soong Y, Seshan SV, Cohen-Gould L, Manichev V, Feldman LC, Gustafsson T. Mitochondria protection after acute ischemia prevents prolonged upregulation of IL-1 $\beta$ and IL-18 and arrests CKD. J Am Soc Nephrol 2017;28(5):1437-49.

8. Zarbock A, Kellum JA, Schmidt C, Van Aken H, Wempe C, Pavenstädt $H$, Boanta A, Gerß J, Meersch M. Effect of early vs delayed initiation of renal replacement therapy on mortality in critically ill patients with acute kidney injury: the ELAIN randomized clinical trial. JAMA 2016;315(20):2190-9.

9. Herbert F, Tchitchek N, Bansal D, Jacques J, Pathak S, Bécavin C, Fesel C, Dalko E, Cazenave PA, Preda C, Ravindran B, Sharma S, Das B, Pied S. Evidence of IL-17, IP-10, and IL-10 involvement in multiple-organ dysfunction and IL-17 pathway in acute renal failure associated to Plasmodium falciparum malaria. J Transl Med 2015;13(1):369.

10. Ozkok A, Ravichandran K, Wang Q, Ljubanovic D, Edelstein CL. NF-KB transcriptional inhibition ameliorates cisplatin-induced acute kidney injury (AKI). Toxicol Lett 2016;240(1):105-13.

11. Khan MM, Yang WL, Brenner M, Bolognese AC, Wang P. Coldinducible RNA-binding protein (CIRP) causes sepsis-associated acute lung injury via induction of endoplasmic reticulum stress. Sci Rep 2017;7:41363.

12. Esposito V, Grosjean F, Tan J, Huang L, Zhu L, Chen J, Xiong H, Striker GE, Zheng F. CHOP deficiency results in elevated lipopolysaccharideinduced inflammation and kidney injury. Am J Physiol Renal Physiol 2012;304(4):F440-50.

13. Calisto KL, Camacho AC, Mittestainer FC, Carvalho BM, Guadagnini D, Carvalheira JB, Saad MJ. RETRACTED ARTICLE: Diacerhein attenuates the inflammatory response and improves survival in a model of severe sepsis. Crit Care 2012;16(4):R158.

14. Ma T, Han L, Gao Y, Li L, Shang X, Hu W, Xue C. The endoplasmic reticulum stress-mediated apoptosis signal pathway is involved in sepsis-induced abnormal lymphocyte apoptosis. Eur Surg Res 2008;41(2):219-25.

15. Toliopoulos IK, Simos YV, Oikonomidis S, Karkabounas SC. Resveratrol diminishes platelet aggregation and increases susceptibility of K562 tumor cells to natural killer cells. Indian J Biochem Biophys 2013;50(1):14-8.

16. Li T, Zhang J, Feng J, Li Q, Wu L, Ye Q, Sun J, Lin Y, Zhang M, Huang R, Cheng J, Cao Y, Xiang G, Zhang J, Wu Q. Resveratrol reduces acute lung injury in a LPS induced sepsis mouse model via activation of Sirt1. Mol Med Rep 2013;7(6):1889-95.

17. Li C, Wang L, Huang K, Zheng L. Endoplasmic reticulum stress in retinal vascular degeneration: protective role of resveratrol. Invest Ophthalmol Vis Sci 2012;53(6):3241-9.

18. Holthoff JH, Wang Z, Seely KA, Gokden N, Mayeux PR. Resveratrol improves renal microcirculation, protects the tubular epithelium, and prolongs survival in a mouse model of sepsis-induced acute kidney injury. Kidney Int 2012;81(4):370-8.

19. Macdonald SP, Williams JM, Shetty A, Bellomo R, Finfer S, Shapiro N, Keijzers G. Review article: Sepsis in the emergency department - Part 
1: Definitions and outcomes. Emerg Med Australas 2017;29(6):61925.

20. Gao X, Fu L, Xiao M, Xu C, Sun L, Zhang T, Zheng F, Mei C. The Nephroprotective Effect of Tauroursodeoxycholic Acid on Ischaemia/ Reperfusion-Induced Acute Kidney Injury by Inhibiting Endoplasmic Reticulum Stress. Basic Clin Pharmacol Toxicol 2012;111(1):14-23.

21. Cybulsky AV. Endoplasmic reticulum stress, the unfolded protein response and autophagy in kidney diseases. Nat Rev Nephrol 2017;13(11):681-96.
22. Tran M, Tam D, Bardia A, Bhasin M, Rowe GC, Kher A, Zsengeller ZK, Akhavan-Sharif MR, Khankin EV, Saintgeniez M, David S, Burstein D, Karumanchi SA, Stillman IE, Arany Z, Parikh SM. PGC-1a promotes recovery after acute kidney injury during systemic inflammation in mice. J Clin Invest 2011;121(10):4003-14.

23. Jung TW, Lee KT, Lee MW, Ka KH. SIRT1 attenuates palmitate-induced endoplasmic reticulum stress and insulin resistance in HepG2 cells via induction of oxygen-regulated protein 150. Biochem Biophys Res Commun 2012;422(2):229-32. 